\title{
Balance Equations of Electromagnetic Angular Momentum
}

\author{
Ignacio Campos-Flores ${ }^{1}$, José Luis Jiménez-Ramírez ${ }^{1}$, José Antonio Eduardo Roa-Neri ${ }^{*}$ \\ ${ }^{1}$ Departamento de Física, Facultad de Ciencias, Universidad Nacional Autónoma de México, Mexico City, México \\ ${ }^{2}$ Área de Física Teórica y Materia Condensada, División de Ciencias Básicas e Ingeniería, Universidad Autónoma Metropolitana \\ Unidad Azcapotzalco, Mexico City, México \\ Email: iecampos@prodigy.net.mx,jlj@xanum.uam.mx, ^rnjae@correo.azc.uam.mx
}

How to cite this paper: Campos-Flores, I., Jiménez-Ramírez, J.L. and Roa-Neri, J.A.E. (2017) Balance Equations of Electromagnetic Angular Momentum. Journal of Electromagnetic Analysis and Applications, 9, 203-217. https://doi.org/10.4236/jemaa.2017.912017

Received: December 7, 2017

Accepted: December 26, 2017

Published: December 29, 2017

Copyright (๑) 2017 by authors and Scientific Research Publishing Inc. This work is licensed under the Creative Commons Attribution International License (CC BY 4.0).

http://creativecommons.org/licenses/by/4.0/

\begin{abstract}
We give theoretical foundation to torque densities proposed in the past, like the one used by Beth to study experimentally the action of circularly polarized radiation on a birefringent material, or that proposed by Mansuripur to resolve a seeming paradox concerning the Lorentz force law and relativity. Our results provide new insights into electromagnetic theory, since they provide a unified and general treatment of the balance of lineal and angular momentum that permits a better assessment of some torques. Thus in this work we extend the derivations we have made of balance equations for electromagnetic linear momentum to balance equations for electromagnetic angular momentum. These balance equations are derived from the macroscopic Maxwell equations by means of vector and tensor identities and from the different ways in which these equations are written in terms of fields $E, D, B$, and $H$, as well as polarizations $P$, and $\boldsymbol{M}$. Therefore these balance equations are as sound as the macroscopic Maxwell equations, with the limitations of the constitutive relations.
\end{abstract}

\section{Keywords}

Balance Equations, Foundations of Electrodynamics, Electromagnetic Angular Momentum, Macroscopic Maxwell Equations, Abraham-Minkowski Controversy

\section{Introduction}

The classical theory of electromagnetism is a well established theory; however, there are still some conceptual problems which deserve insightful reflections, particularly in the theory of electromagnetic media. 
The understanding of the interaction of electromagnetic fields and matter constitutes an open question [1] [2] [3]. There is a debate, initiated by Abraham and Minkowski, about the correct definition of electromagnetic momentum in matter [4] [5] [6] [7]. Besides, some experiments support one definition and other experiments support another. Brevik [8] has recounted some of these proposals and the experiments that support them. We have here, as Brevik [9] notes, a confusing situation, since some experiments support the Helmholtz force density and not the Einstein-Laub [9] force density, while Mansuripur [10] uses the Einstein-Laub force density plus a proposed torque to resolve an apparent inconsistency between the Lorentz force density and relativity. Brevik [8] concludes that "Although arguments of this kind are physically appealing one cannot, however, look upon them as forming a physical derivation". This lack of a coherent derivation from the Maxwell equations has led to controversies like that of Abraham and Minkowski, or paradoxical results as that noted by Mansuripur. Besides this coherent derivation, it is necessary that a coherent interpretation allows resolving these controversial points. We have here a serious unsolved problem where there is still some confusion.

A very fundamental conceptual aspect in Maxwell's theory of electromagnetism is that the electromagnetic field is a kind of generalized mechanical system which has energy, momentum, stresses, and angular momentum. We have then balance equations for energy, momentum, angular momentum and energy-momentumstress. The energy balance is expressed in Poynting's theorem, derived directly as a general integral of Maxwell's equations. The balance equation for electromagnetic momentum, however, is usually derived from the Lorentz force law [11] which implies only an external electromagnetic field. A derivation without this assumption can be found in reference [12]. The momentum balance is usually obtained from Lorentz's force density by substitution of the charge and current densities from the Maxwell equations. This produces a strange mixture of external fields and total fields. We have found [13], however, that several momentum balance equations can also be obtained directly from the macroscopic Maxwell equations by means of vector and tensor identities. These different balance equations result from different ways of expressing the macroscopic Maxwell equations in terms of fields $E, D, B, H$, and polarizations $P$ and $M$. This approach has led to a possible settlement of the old Abraham-Minkowski controversy, since these proposals for the electromagnetic momentum appear in different balance equations [13]. In the present work we use the same approach to derive balance equations of electromagnetic angular momentum, subject that has occupied theorists and experimentalists since long ago, for example Poynting in 1909 [14]. We have also the work of Beth [15], who in 1936 published his famous article on the interaction of circularly polarized light with a birefringent medium. This work is considered the experimental determination of the angular momentum of the photon. The torque proposed by this author, also mentioned by Bohren [16], however, is presented without a derivation. Also, the torque proposed by Man- 
suripur [10] to allege an inconsistency between the Lorentz force and relativity lacks a derivation and is presented only with plausibility arguments. Therefore the equations of balance for either energy or momentum or angular momentum require a logical derivation from the macroscopic Maxwell equations. In the present work we generalize previously obtained balance equations for electromagnetic lineal momentum [12] [13] to balance equations for electromagnetic angular momentum. This means that an insightful reflection on the conceptual structure of classical field theory is necessary to attack these conceptual problems.

In many works on the angular momentum of radiation it is usual to begin either with an expression for the angular momentum of radiation [15] [17] [18], or with an expression for the torque that radiation fields exert on matter [19] [20]. Barnett [21] has derived a balance equation for vacuum, in which the torque used by Beth does not appear, since this torque results from the interaction of radiation with a non-isotropic medium. Cameron et al. [22] also derive a balance equation departing from Noether's theorem, but only for fields in vacuum. We find then a need to derive carefully balance equations of angular momentum of radiation directly from the macroscopic Maxwell equations, as we have done for the linear momentum of radiation [12] [13]. We hope in this way to establish a unified and general view of the balance of electromagnetic angular momentum that permits us to asses torques that have been proposed by some authors. Thus our objective in this work is to deduce several balance equations for angular electromagnetic momentum according to the different expressions that the Maxwell equations acquire in terms of the electromagnetic fields and polarizations. These balance equations have the structure

$$
\nabla \cdot \vec{M}-\partial_{t} \mathbf{I}=\tau,
$$

where $\overrightarrow{\boldsymbol{M}}$ is an angular momentum flux density, $\boldsymbol{I}$ is an angular momentum density and $\tau$ is a torque density, which can then be specialized to obtain particular torques. We hope in this way to give theoretical foundation to the torque proposed by Beth, and to resolve the seeming inconsistency between the Lorentz force and relativity pointed out by Mansuripur [10].

\section{Some Considerations about Several Balance Equations Derived from the Maxwell Equations}

We have shown in past works [12] [13] how from the macroscopic Maxwell equations different momentum balance equations can be deduced by means of vector and tensor identities. These balance equations arise from different forms of expressing the Maxwell equations and constitutive relations in terms of the electromagnetic fields $E, D, B$, and $H$, and the electric and magnetic polarizations $\boldsymbol{P}$ and $\boldsymbol{M}$. We have also that $\boldsymbol{\nabla} \cdot \boldsymbol{P}=-\rho_{\text {pol }}$ and $\boldsymbol{\nabla} \times \boldsymbol{M}=\boldsymbol{J}_{\text {mag }}$. These relations permit us to write the Maxwell equations in many different ways. Here we resume five different ways of expressing the Maxwell equations, with their corresponding momentum balance equations deduced from them. From these bal- 
ance equations we want to obtain angular momentum balance equations which will permit us to analyze the origin of the torque experimentally tested by Beth [15], and the torque which Mansuripur [10] interpreted as an inconsistency between the Lorentz force and the principle of relativity.

We begin with the most usual form of writing the Maxwell equations,

$$
\begin{aligned}
& \nabla \cdot \boldsymbol{D}=\rho, \\
& \nabla \cdot \boldsymbol{B}=0, \\
& \nabla \times \boldsymbol{E}+\partial_{t} \boldsymbol{B}=0, \\
& \nabla \times \boldsymbol{H}-\partial_{t} \boldsymbol{D}=\boldsymbol{J} .
\end{aligned}
$$

The corresponding momentum balance equation deduced from these equations with the aid of vector and tensor identities is

$$
\begin{aligned}
& \nabla \cdot\left\{(\boldsymbol{D E}+\boldsymbol{B H})-\frac{1}{2} \boldsymbol{I}(\boldsymbol{D} \cdot \boldsymbol{E}+\boldsymbol{B} \cdot \boldsymbol{H})\right\}-\partial_{t}(\boldsymbol{D} \times \boldsymbol{B}) \\
& =\rho \boldsymbol{E}+\boldsymbol{J} \times \boldsymbol{B}+\frac{1}{2}[(\nabla \boldsymbol{E}) \cdot \boldsymbol{D}-(\nabla \boldsymbol{D}) \cdot \boldsymbol{E}+(\nabla \boldsymbol{H}) \cdot \boldsymbol{B}-(\nabla \boldsymbol{B}) \cdot \boldsymbol{H}] .
\end{aligned}
$$

This balance equation contains as a particular case the usual Lorentz force; we simply specialize the equation for vacuum and take the limit of a test charge.

Other form of expressing the Maxwell equations, also usual and appearing in the textbook by Panofsky and Phillips [23], is

$$
\begin{aligned}
& \nabla \cdot \boldsymbol{E}=\left(1 / \varepsilon_{0}\right)(\rho-\nabla \cdot \boldsymbol{P}), \\
& \nabla \cdot \boldsymbol{B}=0, \\
& \nabla \times \boldsymbol{E}+\partial_{t} \boldsymbol{B}=\mathbf{0}, \\
& \nabla \times \boldsymbol{B}-\varepsilon_{0} \mu_{0} \partial_{t} \boldsymbol{E}=\mu_{0}\left(\boldsymbol{J}+\partial_{t} \boldsymbol{P}+\nabla \times \boldsymbol{M}\right),
\end{aligned}
$$

whose associated momentum balance equation obtained with the same method is

$$
\begin{aligned}
& \boldsymbol{\nabla} \cdot\left\{\left(\varepsilon_{0} \boldsymbol{E} \boldsymbol{E}+\left(\frac{1}{\mu_{0}}\right) \boldsymbol{B} \boldsymbol{B}\right)-\frac{1}{2} \boldsymbol{I}\left(\varepsilon_{0} \boldsymbol{E} \cdot \boldsymbol{E}+\left(\frac{1}{\mu_{0}}\right) \boldsymbol{B} \cdot \boldsymbol{B}\right)\right\}-\varepsilon_{0} \partial_{t}(\boldsymbol{E} \times \boldsymbol{B}) \\
& =\rho \boldsymbol{E}+\boldsymbol{J} \times \boldsymbol{B}-(\nabla \cdot \boldsymbol{P}) \boldsymbol{E}+\left(\partial_{t} \boldsymbol{P}\right) \times \boldsymbol{B}+(\nabla \times \boldsymbol{M}) \times \boldsymbol{B} .
\end{aligned}
$$

Again, the Lorentz force density appears as a particular case.

A third way of expressing the Maxwell equations is

$$
\begin{aligned}
& \nabla \cdot \boldsymbol{D}=\rho, \\
& \nabla \cdot \boldsymbol{B}=0, \\
& \nabla \times \boldsymbol{E}+\mu_{0} \partial_{t} \boldsymbol{H}=-\mu_{0} \partial_{t} \boldsymbol{M}, \\
& \nabla \times \boldsymbol{H}-\varepsilon_{0} \partial_{t} \boldsymbol{E}=\boldsymbol{J}+\partial_{t} \boldsymbol{P},
\end{aligned}
$$

with an associated momentum balance equation given by

$$
\begin{aligned}
\nabla \cdot\left\{(\boldsymbol{D E}+\boldsymbol{B H})-\frac{1}{2} \boldsymbol{I}\left(\varepsilon_{0} \boldsymbol{E} \cdot \boldsymbol{E}+\mu_{0} \boldsymbol{H} \cdot \boldsymbol{H}\right)\right\}-\varepsilon_{0} \mu_{0} \partial_{t}(\boldsymbol{E} \times \boldsymbol{H}) \\
=\rho \boldsymbol{E}+\mu_{0} \boldsymbol{J} \times \boldsymbol{H}+(\boldsymbol{P} \cdot \nabla) \boldsymbol{E}+\mu_{0}\left(\partial_{t} \boldsymbol{P}\right) \times \boldsymbol{H} \\
-\varepsilon_{0} \mu_{0}\left(\partial_{t} \boldsymbol{M}\right) \times \boldsymbol{E}+\mu_{0}(\boldsymbol{M} \cdot \nabla) \boldsymbol{H} .
\end{aligned}
$$

If fields $\boldsymbol{D}$ and $\boldsymbol{B}$ are considered as the fundamental fields, then the Maxwell 
equations may be expressed in the form

$$
\begin{aligned}
& \nabla \cdot \boldsymbol{D}=\rho, \\
& \nabla \cdot \boldsymbol{B}=0, \\
& \nabla \times \boldsymbol{D}+\varepsilon_{0} \partial_{t} \boldsymbol{B}=\nabla \times \boldsymbol{P}, \\
& \nabla \times \boldsymbol{B}-\mu_{0} \partial_{t} \boldsymbol{D}=\mu_{0} \boldsymbol{J}+\mu_{0} \boldsymbol{\nabla} \times \boldsymbol{M},
\end{aligned}
$$

and the associated momentum balance equation results

$$
\begin{aligned}
& \nabla \cdot\left\{\left(\frac{1}{\varepsilon_{0}} \boldsymbol{D D}+\frac{1}{\mu_{0}} \boldsymbol{B} \boldsymbol{B}\right)-\frac{1}{2} \boldsymbol{I}\left(\frac{1}{\varepsilon_{0}} \boldsymbol{D} \cdot \boldsymbol{D}+\frac{1}{\mu_{0}} \boldsymbol{B} \cdot \boldsymbol{B}\right)\right\}-\partial_{t}(\boldsymbol{D} \times \boldsymbol{B}) \\
& =\frac{1}{\varepsilon_{0}} \rho \boldsymbol{D}+\boldsymbol{J} \times \boldsymbol{B}+\frac{1}{\varepsilon_{0}}(\nabla \times \boldsymbol{P}) \times \boldsymbol{D}+(\nabla \times \boldsymbol{M}) \times \boldsymbol{B} .
\end{aligned}
$$

We have yet other form of expressing the Maxwell Equations, which is

$$
\begin{aligned}
& \nabla \cdot \boldsymbol{E}=\left(1 / \varepsilon_{0}\right)(\rho-\nabla \cdot \boldsymbol{P}), \\
& \nabla \cdot \boldsymbol{H}=-\nabla \cdot \boldsymbol{M}, \\
& \nabla \times \boldsymbol{E}+\mu_{0} \partial_{t} \boldsymbol{H}=-\mu_{0} \partial_{t} \boldsymbol{M}, \\
& \nabla \times \boldsymbol{H}-\varepsilon_{0} \partial_{t} \boldsymbol{E}=\boldsymbol{J}+\partial_{t} \boldsymbol{P},
\end{aligned}
$$

with the following balance equation

$$
\begin{aligned}
\nabla \cdot\left\{\left(\varepsilon_{0} \boldsymbol{E} \boldsymbol{E}+\mu_{0} \boldsymbol{H H}\right)-\frac{1}{2} \boldsymbol{I}\left(\varepsilon_{0} \boldsymbol{E} \cdot \boldsymbol{E}+\mu_{0} \boldsymbol{H} \cdot \boldsymbol{H}\right)\right\}-\varepsilon_{0} \mu_{0} \partial_{t}(\boldsymbol{E} \times \boldsymbol{H}) \\
=\rho \boldsymbol{E}+\mu_{0} \boldsymbol{J} \times \boldsymbol{H}-(\nabla \cdot \boldsymbol{P}) \boldsymbol{E}-\mu_{0}(\nabla \cdot \boldsymbol{M}) \boldsymbol{H} \\
+\mu_{0}\left(\partial_{t} \boldsymbol{P}\right) \times \boldsymbol{H}+\varepsilon_{0} \mu_{0} \boldsymbol{E} \times\left(\partial_{t} \boldsymbol{M}\right) .
\end{aligned}
$$

There are other forms of expressing the Maxwell equations, but these five different ways of expressing these equations are sufficient to illustrate the method for deducing now balance equations of electromagnetic angular momentum, and for the analysis of the torque used by Beth [15] and that considered by Mansuripur [10].

\section{Balance Equations of Angular Momentum}

We can observe that all the above balance equations have the structure

$$
\nabla \cdot \overrightarrow{\boldsymbol{T}}-\partial_{t} \boldsymbol{g}=\boldsymbol{f}_{L}+\delta \boldsymbol{f}
$$

where the tensor or dyad $\overrightarrow{\boldsymbol{T}}$ has the form

$$
\overleftrightarrow{\boldsymbol{T}}=\overleftrightarrow{\boldsymbol{T}}_{i}-\boldsymbol{I} u_{i}
$$

(sub index $i$ refers to the different tensors that can be constructed) while $\boldsymbol{f}_{L}+\delta \boldsymbol{f}$ are force densities; $\boldsymbol{f}_{L}$ is a force density analogous to that of Lorentz, which involves charge and current densities, and $\delta \boldsymbol{f}$ is a force involving only fields [12] [13].

The tensor $\overrightarrow{\boldsymbol{T}}_{i}$ is the addition of dyads of type $\boldsymbol{F} \boldsymbol{G}$, where $\boldsymbol{F}$ and $\boldsymbol{G}$ are either electric vectors (for example $\boldsymbol{E D}$ ) or magnetic vectors (for example $\boldsymbol{B H}$ ), while $u_{i}$ is an expression of type energy density, that is, the scalar product of electric vectors plus the scalar product of magnetic vectors; $\boldsymbol{g}$ is the vector product of an 
electric vector and a magnetic vector with units of momentum density (as the Poynting vector divided by $c^{2}$ ). With this general scheme we can analyze all the above balance equations.

Before beginning the derivation of balance equations of angular momentum it is necessary to note that in the above balance equations we have considered only isotropic media. However, if we want to analyze experiments like the one performed by Beth [15], where effects associated to the angular momentum of radiation are measured, we find necessary to consider non isotropic media. Therefore it also becomes necessary to separate the tensors involved in Equations (3), (5), (7), (9) and (11) into its symmetrical and anti-symmetrical parts. This will be necessary only for the tensor $\overrightarrow{\boldsymbol{T}}_{i}$, since the other part is symmetrical.

Then we have

$$
\overrightarrow{\boldsymbol{T}}_{i}=(1 / 2)(\boldsymbol{F} \boldsymbol{G}+\boldsymbol{G F})+(1 / 2)(\boldsymbol{F} \boldsymbol{G}-\boldsymbol{G} \boldsymbol{F}),
$$

where the symmetrical and anti-symmetrical parts of the tensor $\overrightarrow{\boldsymbol{T}}$ are defined by

$$
\left.\ddot{\boldsymbol{T}}^{S}\right|_{i}=(1 / 2)(\boldsymbol{F} \boldsymbol{G}+\boldsymbol{G F})-\boldsymbol{I} u_{i}
$$

and

$$
\left.\overrightarrow{\mathbf{T}}^{A}\right|_{i}=(1 / 2)(\boldsymbol{F} \boldsymbol{G}-\boldsymbol{G F}),
$$

respectively. With this separation we can write the general balance Equation (11) as

$$
\nabla \cdot\left(\left.\ddot{\boldsymbol{T}}^{S}\right|_{i}+\left.\ddot{\boldsymbol{T}}^{A}\right|_{i}\right)-\partial_{t} \boldsymbol{g}=\boldsymbol{f}_{L}+\delta \boldsymbol{f}
$$

In order to obtain a general balance equation of angular momentum we multiply vectorially this equation by $-\boldsymbol{r}$ on the right, getting

$$
-\nabla \cdot\left(\left.\ddot{\boldsymbol{T}}^{S}\right|_{i}+\left.\overrightarrow{\boldsymbol{T}}^{A}\right|_{i}\right) \times \boldsymbol{r}-\partial_{t}(\boldsymbol{r} \times \boldsymbol{g})=\boldsymbol{r} \times\left(\boldsymbol{f}_{L}+\delta \boldsymbol{f}\right) .
$$

Now we want to rewrite the first term on the left as a divergence of a tensor which looks like $\nabla \cdot(\overrightarrow{\boldsymbol{T}} \times \boldsymbol{r})$, and thus it is necessary to find a relation between $\boldsymbol{\nabla} \cdot\left(\left.\ddot{\boldsymbol{T}}^{S}\right|_{i}+\left.\overrightarrow{\boldsymbol{T}}^{A}\right|_{i}\right) \times \boldsymbol{r}$ and $\nabla \cdot\left[\left(\left.\ddot{\boldsymbol{T}}^{S}\right|_{i}+\left.\overrightarrow{\boldsymbol{T}}^{A}\right|_{i}\right) \times \boldsymbol{r}\right]$. We proceed as follows.

Given the general form of the tensor $\overrightarrow{\boldsymbol{T}}_{i}$ it is necessary to analyze only a second rank tensor of type $F G$, and then to construct its symmetrical and anti-symmetrical parts.

Then by means of the identity

$$
\nabla \cdot[\boldsymbol{F} \boldsymbol{\eta}]=(\boldsymbol{\nabla} \cdot \boldsymbol{F}) \boldsymbol{\eta}+(\boldsymbol{F} \cdot \nabla) \boldsymbol{\eta}
$$

we obtain

$$
(\nabla \cdot \boldsymbol{F} \boldsymbol{G}) \times \boldsymbol{r}=(\nabla \cdot \boldsymbol{F}) \boldsymbol{G} \times \boldsymbol{r}+[(\boldsymbol{F} \cdot \nabla) \boldsymbol{G}] \times \boldsymbol{r} .
$$

But we have that

$$
(\boldsymbol{F} \cdot \nabla)(\boldsymbol{G} \times \boldsymbol{r})=[(\boldsymbol{F} \cdot \nabla) \boldsymbol{G}] \times \boldsymbol{r}+\boldsymbol{G} \times \boldsymbol{F}
$$


since $(\boldsymbol{F} \cdot \nabla) \boldsymbol{r}=\boldsymbol{F}$, then

$$
(\nabla \cdot \boldsymbol{F} \boldsymbol{G}) \times \boldsymbol{r}=(\nabla \cdot \boldsymbol{F}) \boldsymbol{G} \times \boldsymbol{r}+(\boldsymbol{F} \cdot \nabla)(\boldsymbol{G} \times \boldsymbol{r})-\boldsymbol{G} \times \boldsymbol{F} .
$$

On the other hand, by using identity (18) we get

$$
\nabla \cdot[\boldsymbol{F}(\boldsymbol{G} \times \boldsymbol{r})]=(\boldsymbol{\nabla} \cdot \boldsymbol{F})(\boldsymbol{G} \times \boldsymbol{r})+(\boldsymbol{F} \cdot \nabla)(\boldsymbol{G} \times \boldsymbol{r}),
$$

from which it follows that

$$
(\boldsymbol{F} \cdot \nabla)(\boldsymbol{G} \times \boldsymbol{r})=\nabla \cdot[\boldsymbol{F}(\boldsymbol{G} \times \boldsymbol{r})]-(\nabla \cdot \boldsymbol{F})(\boldsymbol{G} \times \boldsymbol{r}) .
$$

Substituting this result in Equation (21) we obtain

$$
(\nabla \cdot F \boldsymbol{G}) \times \boldsymbol{r}=\nabla \cdot[\boldsymbol{F}(\boldsymbol{G} \times \boldsymbol{r})]-\boldsymbol{G} \times \boldsymbol{F},
$$

which is the result we were looking for.

This equation can be expressed in terms of a dual tensor, defined as

$$
T_{i j}^{d}=\theta_{k}=(1 / 2) \epsilon_{k i j} T_{i j} .
$$

Then, if $T_{i j}=F_{i} G_{j}$, we have

$$
\boldsymbol{F} \times \boldsymbol{G}=2 \ddot{\boldsymbol{T}}^{d} .
$$

Using now the results expressed in Equations (24) and (26) permits to rewrite the left-hand member of Equation (17) as

$$
\left[\nabla \cdot\left(\left.\ddot{\boldsymbol{T}}^{S}\right|_{i}+\left.\ddot{\boldsymbol{T}}^{A}\right|_{i}\right)\right] \times \boldsymbol{r}=\nabla \cdot\left\{\left(\left.\overrightarrow{\boldsymbol{T}}^{S}\right|_{i}+\left.\overrightarrow{\boldsymbol{T}}^{A}\right|_{i}\right) \times \boldsymbol{r}\right\}+2 \overrightarrow{\boldsymbol{T}}^{d} .
$$

Then Equation (17) can be written in the form

$$
\nabla \cdot \overrightarrow{\boldsymbol{M}}-\partial_{t} \boldsymbol{I}=\left(\tau_{L}+\delta \tau\right)+2 \overrightarrow{\boldsymbol{T}}^{d},
$$

where

$$
\begin{aligned}
& \overrightarrow{\boldsymbol{M}}=-\left.\overrightarrow{\boldsymbol{T}}\right|_{i} \times \boldsymbol{r}, \\
& \boldsymbol{I}=\boldsymbol{r} \times \boldsymbol{g}, \\
& \tau_{L}=\boldsymbol{r} \times \boldsymbol{f}_{L}, \\
& \delta \boldsymbol{\tau}=\boldsymbol{r} \times \delta \boldsymbol{f} .
\end{aligned}
$$

It is important to note that the general balance equation contains a new torque of type $\boldsymbol{F} \times \boldsymbol{G}$, exactly of the form considered by Beth [15], relevant only for non isotropic media, that here has emerged from a general balance equation implied by the macroscopic Maxwell equations.

We can observe that in the balance Equations (3), (5), (7), (9) and (11) only Equations (3) and (7) contain a non-symmetrical tensor (in fact the same tensor). The other equations contain different but symmetrical tensors. In a vacuum they are equal, and then in this case the balance equations of angular momentum do not contain terms of type $\boldsymbol{F} \times \boldsymbol{G}$.

Now, it is possible to obtain directly the balance equations of angular momentum from Equation (29) and Equations (3), (5), (7), (8) and (11). In order to express them more compactly it is convenient to define the tensors 


$$
\begin{aligned}
\overrightarrow{\boldsymbol{T}}_{1}= & \left(\frac{1}{2}\right)[(\boldsymbol{D} \boldsymbol{E}+\boldsymbol{B H})+(\boldsymbol{E} \boldsymbol{D}+\boldsymbol{H B})]+\left(\frac{1}{2}\right)[(\boldsymbol{D E}+\boldsymbol{B H})-(\boldsymbol{E D}+\boldsymbol{H B})] \\
& -\frac{1}{2} \boldsymbol{I}(\boldsymbol{D} \cdot \boldsymbol{E}+\boldsymbol{B} \cdot \boldsymbol{H}), \\
\overrightarrow{\boldsymbol{T}}_{2}= & \varepsilon_{0} \boldsymbol{E} \boldsymbol{E}+\left(\frac{1}{\mu_{0}}\right) \boldsymbol{B B}-\frac{1}{2} \boldsymbol{I}\left(\varepsilon_{0} \boldsymbol{E} \cdot \boldsymbol{E}+\left(\frac{1}{\mu_{0}}\right) \boldsymbol{B} \cdot \boldsymbol{B}\right), \\
\overrightarrow{\boldsymbol{T}}_{3}= & \left(\frac{1}{2}\right)[(\boldsymbol{D} \boldsymbol{E}+\boldsymbol{B H})+(\boldsymbol{E} \boldsymbol{D}+\boldsymbol{H B})]+\left(\frac{1}{2}\right)[(\boldsymbol{D E}+\boldsymbol{B H})-(\boldsymbol{E D}+\boldsymbol{H B})] \\
& -\frac{1}{2} \boldsymbol{I}\left(\varepsilon_{0} \boldsymbol{E} \cdot \boldsymbol{E}+\mu_{0} \boldsymbol{H} \cdot \boldsymbol{H}\right), \\
\overrightarrow{\boldsymbol{T}}_{4}= & \frac{1}{\varepsilon_{0}} \boldsymbol{D D}+\frac{1}{\mu_{0}} \boldsymbol{B B}-\frac{1}{2} \boldsymbol{I}\left(\frac{1}{\varepsilon_{0}} \boldsymbol{D} \cdot \boldsymbol{D}+\frac{1}{\mu_{0}} \boldsymbol{B} \cdot \boldsymbol{B}\right)
\end{aligned}
$$

and

$$
\overleftrightarrow{\boldsymbol{T}}_{5}=\varepsilon_{0} \boldsymbol{E} \boldsymbol{E}+\mu_{0} \boldsymbol{H H}-\frac{1}{2} \boldsymbol{I}\left(\varepsilon_{0} \boldsymbol{E} \cdot \boldsymbol{E}+\mu_{0} \boldsymbol{H} \cdot \boldsymbol{H}\right) .
$$

With these tensors the balance equations can be expressed in the form

$$
\begin{aligned}
- & \nabla \cdot\left(\ddot{\boldsymbol{T}}_{1} \times \boldsymbol{r}\right)-(\boldsymbol{D} \times \boldsymbol{E}+\boldsymbol{B} \times \boldsymbol{H})-\partial_{t}(\boldsymbol{r} \times(\boldsymbol{D} \times \boldsymbol{B})) \\
= & \boldsymbol{r} \times\left(\rho \boldsymbol{E}+\boldsymbol{J} \times \boldsymbol{B}+\frac{1}{2}[(\nabla \boldsymbol{E}) \cdot \boldsymbol{D}-(\nabla \boldsymbol{D}) \cdot \boldsymbol{E}+(\nabla \boldsymbol{B}) \cdot \boldsymbol{H}-(\nabla \boldsymbol{H}) \cdot \boldsymbol{B}]\right), \\
- & \nabla \cdot\left(\overrightarrow{\boldsymbol{T}}_{2} \times \boldsymbol{r}\right)-\partial_{t} \boldsymbol{r} \times\left(\varepsilon_{0} \boldsymbol{E} \times \boldsymbol{B}\right) \\
= & \boldsymbol{r} \times\left(\rho \boldsymbol{E}+\boldsymbol{J} \times \boldsymbol{B}-(\nabla \cdot \boldsymbol{P}) \boldsymbol{E}+\left(\partial_{t} \boldsymbol{P}\right) \times \boldsymbol{B}+(\nabla \times \boldsymbol{M}) \times \boldsymbol{B}\right), \\
- & \nabla \cdot\left(\overrightarrow{\boldsymbol{T}}_{3} \times \boldsymbol{r}\right)-(\boldsymbol{D} \times \boldsymbol{E}+\boldsymbol{B} \times \boldsymbol{H})-\partial_{t}\left(\boldsymbol{r} \times\left(\varepsilon_{0} \mu_{0} \boldsymbol{E} \times \boldsymbol{H}\right)\right) \\
= & \boldsymbol{r} \times\left(\rho \boldsymbol{E}+\mu_{0} \boldsymbol{J} \times \boldsymbol{H}+(\boldsymbol{P} \cdot \nabla) \boldsymbol{E}+\mu_{0}\left(\partial_{t} \boldsymbol{P}\right) \times \boldsymbol{H}\right. \\
& \left.-\varepsilon_{0} \mu_{0}\left(\partial_{t} \boldsymbol{M}\right) \times \boldsymbol{E}+\mu_{0}(\boldsymbol{M} \cdot \nabla) \boldsymbol{H}\right), \\
- & \nabla \cdot\left(\overrightarrow{\boldsymbol{T}}_{4} \times \boldsymbol{r}\right)-\partial_{t}(\boldsymbol{r} \times(\boldsymbol{D} \times \boldsymbol{B})) \\
= & \boldsymbol{r} \times\left(\frac{1}{\varepsilon_{0}} \rho \boldsymbol{D}+\boldsymbol{J} \times \boldsymbol{B}+\frac{1}{\varepsilon_{0}}(\nabla \times \boldsymbol{P}) \times \boldsymbol{D}+(\nabla \times \boldsymbol{M}) \times \boldsymbol{B}\right) \\
- & \nabla \cdot\left(\overrightarrow{\boldsymbol{T}}_{5} \times \boldsymbol{r}\right)-\partial_{t}\left(\boldsymbol{r} \times\left(\varepsilon_{0} \mu_{0} \boldsymbol{E} \times \boldsymbol{H}\right)\right) \\
= & \boldsymbol{r} \times\left(\rho \boldsymbol{E}+\mu_{0} \boldsymbol{J} \times \boldsymbol{H}-(\nabla \cdot \boldsymbol{P}) \boldsymbol{E}-\mu_{0}(\nabla \cdot \boldsymbol{M}) \boldsymbol{H}\right. \\
& \left.+\mu_{0}\left(\partial_{t} \boldsymbol{P}\right) \times \boldsymbol{H}+\varepsilon_{0} \mu_{0} \boldsymbol{E} \times\left(\partial_{t} \boldsymbol{M}\right)\right) .
\end{aligned}
$$

We have in this way the desired balance equations. We have obtained them directly from the Maxwell equations through the balance equations of electromagnetic lineal momentum, and therefore they are all as sound as the Maxwell equations. What equation is convenient to use depends on the particular physical problem we want to solve. For example, if we want to study the interaction between radiation and a non isotropic media, then we have only Equations (35) and (37) as tools for the analysis of the problem. Thus a problem will require, or 
will be simpler, if the convenient fields and polarizations are used.

\section{Some Comments about the Applicability of the Balance Equations of Angular Momentum}

As we mentioned in the introduction, the usual way of trying to obtain a momentum balance equation is through the density force, expressed in the Lorentz force density [4] [23]. Thus terms representing charge and current densities, such as $\boldsymbol{\nabla} \cdot \boldsymbol{P}=-\rho_{\text {pol }}$ and $\boldsymbol{\nabla} \times \boldsymbol{M}=\boldsymbol{J}_{\text {mag }}$, are tentatively added to the Lorentz force density, taking this law as a postulate independent of the Maxwell equations [10]. However, we have shown [12] [13] that it is possible to cast the Maxwell equations into momentum balance equations. This method shows how different electromagnetic momentum and force densities arise, which can be considered as equivalent, since they are implications of the Maxwell equations.

In the present case we have that the angular momentum densities associated to each of the obtained balance equations are

$$
\begin{aligned}
& \boldsymbol{l}_{1}=\boldsymbol{r} \times(\boldsymbol{D} \times \boldsymbol{B}) \\
& \boldsymbol{I}_{2}=\boldsymbol{r} \times\left(\varepsilon_{0} \boldsymbol{E} \times \boldsymbol{B}\right) \\
& \boldsymbol{I}_{3}=\boldsymbol{r} \times\left(\varepsilon_{0} \mu_{0} \boldsymbol{E} \times \boldsymbol{H}\right) \\
& \boldsymbol{I}_{4}=\boldsymbol{r} \times(\boldsymbol{D} \times \boldsymbol{B}) \\
& \boldsymbol{I}_{5}=\boldsymbol{r} \times\left(\varepsilon_{0} \mu_{0} \boldsymbol{E} \times \boldsymbol{H}\right) .
\end{aligned}
$$

It is interesting that, $\boldsymbol{l}_{1}=\boldsymbol{l}_{4}$, consistent with Minkoski's proposal for the definition of the electromagnetic momentum density, while $\boldsymbol{I}_{3}=\boldsymbol{I}_{5}$, consistent with Abraham's definition of electromagnetic momentum density. However, $\boldsymbol{I}_{2}$ is not associated with any of these two proposals. Again, we see that the Abraham-Minkowski controversy arises from considering their proposals without considering the balance equations to which they pertain, observing that there are other possible momentum densities.

We emphasize that the balance equations obtained, both of lineal and angular momentum, are as general as the Maxwell equations, from which they are derived by means of vector and tensor identities. In this way our approach permits to obtain, by taking particular or limit cases of the balance equations, different proposals of electromagnetic lineal and angular momentum densities and others that would contain $\boldsymbol{P} \times \boldsymbol{B}$, as well as the force and torque densities. For example, all the balance equations of lineal momentum have in the limit of a test charge and for a vacuum the usual Lorentz force density, which is taken in the usual approach as a postulate, independent of the Maxwell equations, and as the point of departure to propose force and torque densities. We can then examine some of the proposed balance equations, or proposed lineal and angular momentum densities, or force and torque densities, as follow.

\section{1) Jackson's proposal of a balance equation}

Since Jackson's text [11] is one that considers a balance equation in its proposed problems (problems 6.9 and 6.10), it is convenient to discuss his proposal, 
which is

$$
\nabla \cdot \overrightarrow{\boldsymbol{M}}^{\prime}-\partial_{t}\left(\boldsymbol{I}_{\text {mech }}+\boldsymbol{I}_{\text {elm }}\right)=0
$$

where

$$
\begin{aligned}
\overrightarrow{\boldsymbol{M}}^{\prime} & =\ddot{\boldsymbol{T}} \times \boldsymbol{r}, \\
\boldsymbol{I}_{e l m} & =\boldsymbol{r} \times \boldsymbol{g}^{\prime} .
\end{aligned}
$$

Here $\overrightarrow{\boldsymbol{T}}$ is Maxwell's tensor,

$$
\overrightarrow{\boldsymbol{T}}=\boldsymbol{D E}+\boldsymbol{B H}-\frac{1}{2} \boldsymbol{I}(\boldsymbol{D} \cdot \boldsymbol{E}+\boldsymbol{B} \cdot \boldsymbol{H}),
$$

and $\boldsymbol{g}^{\prime}$ is the lineal momentum density proposed by Minkowski,

$$
\boldsymbol{g}^{\prime}=\boldsymbol{D} \times \boldsymbol{B} .
$$

However, Jackson considers only homogeneous and isotropic media, so that it seems that the balance equations of lineal and angular momentums are almost identical with those for vacuum, that is, they have the structure of Equation (41). If we compare Jackson's proposal with one of the balance equations obtained with our approach, we could identify it with Equation (35) for the case in which there are not free charge and current densities, but we note that the term

$$
\delta \boldsymbol{f}=\frac{1}{2}[(\nabla \boldsymbol{E}) \cdot \boldsymbol{D}-(\nabla \boldsymbol{D}) \cdot \boldsymbol{E}+(\nabla \boldsymbol{H}) \cdot \boldsymbol{B}-(\nabla \boldsymbol{B}) \cdot \boldsymbol{H}]
$$

is absent. This means that this balance equation is inappropriate to deal with force densities that arise in treating inhomogeneous media, since it lacks the Helmholtz force density, the radiation force, or the torque on birefringent media considered by Beth [15]. On the other hand, the text by Panofsky and Phillips [23] does consider a balance equation of lineal momentum for inhomogeneous media, but unfortunately does not consider the balance of angular momentum nor anisotropic media.

It important to note that the balance equations of the form of Equation (41) are valid only for vacuum, or for media of constant permittivity and permeability. The balance equations obtained with our method are more general and more complex, the extra terms being relevant for the cases mentioned above.

\section{2) Balance equation to understand Beth's work}

In his work Beth considers a torque $\boldsymbol{P} \times \boldsymbol{E}$, without indicating where it comes from. This torque is also mentioned in Bohren's work [16], but again without indicating its origin. In the balance equations here described only Equation (35) and Equation (37) contain the term $\boldsymbol{D} \times \boldsymbol{E}+\boldsymbol{B} \times \boldsymbol{H}$, resulting from the fact that non symmetrical tensors are involved in the balance equations. This again indicates that it is the particular physical problem to solve what imposes the balance equation to use.

Since both are equivalent, we will use Equation (35) without free charge or current densities. Then Equation (35) in this case is 


$$
\begin{aligned}
& -\nabla \cdot\left(\overrightarrow{\boldsymbol{T}}_{1} \times \boldsymbol{r}\right)-(\boldsymbol{D} \times \boldsymbol{E}+\boldsymbol{B} \times \boldsymbol{H})-\partial_{t}(\boldsymbol{r} \times(\boldsymbol{D} \times \boldsymbol{B})) \\
& =\boldsymbol{r} \times \frac{1}{2}((\nabla \boldsymbol{E}) \cdot \boldsymbol{D}-(\nabla \boldsymbol{D}) \cdot \boldsymbol{E}+(\nabla \boldsymbol{H}) \cdot \boldsymbol{B}-(\nabla \boldsymbol{B}) \cdot \boldsymbol{H}),
\end{aligned}
$$

where the tensor $\overrightarrow{\boldsymbol{T}}_{1}^{\prime}$ is defined as

$$
\overrightarrow{\boldsymbol{T}}_{1}^{\prime}=\left(\frac{1}{2}\right)[(\boldsymbol{D E}+\boldsymbol{B H})+(\boldsymbol{E D}+\boldsymbol{H B})]+\left(\frac{1}{2}\right)[\boldsymbol{D E}-\boldsymbol{E D}]-\frac{1}{2} \boldsymbol{I}(\boldsymbol{D} \cdot \boldsymbol{E}+\boldsymbol{B} \cdot \boldsymbol{H}) .
$$

It is enough to express it as

$$
\ddot{\boldsymbol{T}}_{1}^{\prime}=\boldsymbol{D E}+\boldsymbol{B H}-\frac{1}{2} \boldsymbol{I}(\boldsymbol{D} \cdot \boldsymbol{E}+\boldsymbol{B} \cdot \boldsymbol{H}) .
$$

In the present case we note that, with the aid of the constitutive relations, we can write

$$
\boldsymbol{D} \times \boldsymbol{E}+\boldsymbol{B} \times \boldsymbol{H}=\boldsymbol{P} \times \boldsymbol{E}+\mu_{0} \boldsymbol{M} \times \boldsymbol{H}
$$

which for non magnetic media reduces to

$$
\boldsymbol{D} \times \boldsymbol{E}+\boldsymbol{B} \times \boldsymbol{H}=\boldsymbol{P} \times \boldsymbol{E}=\tau_{\text {Beth }},
$$

where $\tau_{\text {Beth }}$ is the torque density proposed by Beth and the tensor $\boldsymbol{T}_{1}^{\prime}$ now is

$$
\ddot{\boldsymbol{T}}_{1 \text { non mag }}^{\prime}=\boldsymbol{D E}-\frac{1}{2} \boldsymbol{I}(\boldsymbol{D} \cdot \boldsymbol{E}) .
$$

Then, for non magnetic media Equation (35) becomes

$$
\begin{aligned}
& -\tilde{\boldsymbol{N}} \cdot\left(\overrightarrow{\boldsymbol{T}}_{1 \text { non mag }}^{\prime} \times \boldsymbol{r}\right)-(\boldsymbol{P} \times \boldsymbol{E})-\partial_{t}(\boldsymbol{r} \times(\boldsymbol{D} \times \boldsymbol{B})) \\
& =\boldsymbol{r} \times \frac{1}{2}((\nabla \boldsymbol{E}) \cdot \boldsymbol{D}-(\boldsymbol{\nabla} \boldsymbol{D}) \cdot \boldsymbol{E}),
\end{aligned}
$$

while the integration of Equation (50), with the tensor (51), over a volume $V$ is

$$
\begin{aligned}
& \int \mathrm{d}^{3} r\left(-\nabla \cdot\left\{\left[\boldsymbol{D E}-\frac{1}{2} \boldsymbol{I}(\boldsymbol{D} \cdot \boldsymbol{E})\right] \times \boldsymbol{r}\right\}\right)+\int \mathrm{d}^{3} r\left(-(\boldsymbol{P} \times \boldsymbol{E})-\partial_{t}(\boldsymbol{r} \times(\boldsymbol{D} \times \boldsymbol{B}))\right) \\
& =\int \mathrm{d}^{3} r\left(\boldsymbol{r} \times \frac{1}{2}((\nabla \boldsymbol{E}) \cdot \boldsymbol{D}-(\boldsymbol{\nabla} \boldsymbol{D}) \cdot \boldsymbol{E})\right) .
\end{aligned}
$$

Gauss's theorem permits us to change the volume integration by a surface integration, resulting

$$
\begin{aligned}
& \oint\left(-\mathrm{d} \boldsymbol{\sigma} \cdot\left\{\left[\boldsymbol{D} \boldsymbol{E}-\frac{1}{2} \boldsymbol{I}(\boldsymbol{D} \cdot \boldsymbol{E})\right] \times \boldsymbol{r}\right\}\right)+\int \mathrm{d}^{3} r\left(-(\boldsymbol{P} \times \boldsymbol{E})-\partial_{t}(\boldsymbol{r} \times(\boldsymbol{D} \times \boldsymbol{B}))\right) \\
& =\int \mathrm{d}^{3} r\left(\boldsymbol{r} \times \frac{1}{2}((\nabla \boldsymbol{E}) \cdot \boldsymbol{D}-(\boldsymbol{\nabla} \boldsymbol{D}) \cdot \boldsymbol{E})\right) .
\end{aligned}
$$

We observe that there appear integrals of type

$$
\oint \mathrm{d} \sigma \cdot \boldsymbol{F} \boldsymbol{G} \times \boldsymbol{r}
$$

and

$$
\oint \mathrm{d} \boldsymbol{\sigma} \cdot \mathbf{I} u \times \boldsymbol{r} .
$$

In order to make the integration easier we can select the integration surface so 
that

$$
\mathrm{d} \sigma=(\mathrm{d} \sigma) \hat{\boldsymbol{k}}
$$

where $\hat{\boldsymbol{k}}$ is a unitary vector in the $z$ direction. Now we use the fact that an electromagnetic wave of finite transversal section has components in the propagation direction, and then in our case a wave has a radial component as well as a component in the propagation direction. Therefore we can write

$$
\boldsymbol{F} \boldsymbol{G}=\left(F_{r} \hat{\boldsymbol{r}}+F_{z} \hat{\boldsymbol{k}}\right)\left(G_{r} \hat{\boldsymbol{r}}+G_{z} \hat{\boldsymbol{k}}\right)
$$

and the integrand is of type

$$
\hat{\boldsymbol{k}} \cdot\left[\left(F_{r} \hat{\boldsymbol{r}}+F_{z} \hat{\boldsymbol{k}}\right)\left(G_{r} \hat{\boldsymbol{r}}+G_{z} \hat{\boldsymbol{k}}\right)\right] \times \boldsymbol{r}=F_{z} G_{z} \hat{\boldsymbol{\varphi}}
$$

On the other hand we have that

$$
\hat{\boldsymbol{k}} \cdot(\boldsymbol{I} u) \times \boldsymbol{r}=u \hat{\boldsymbol{\varphi}}
$$

also in direction $\hat{\varphi}$. The implications of these results is that there is no contribution to the transfer of angular momentum due to the tensor $\boldsymbol{T}_{1}$, so that the balance of angular momentum becomes

$$
-\int \mathrm{d}^{3} r \partial_{t}(\boldsymbol{r} \times(\boldsymbol{D} \times \boldsymbol{B}))=\int \mathrm{d}^{3} r(\boldsymbol{P} \times \boldsymbol{E})
$$

or equivalently, for a wave incident from vacuum, to

$$
-\int \mathrm{d}^{3} r \varepsilon_{0} \partial_{t}(\boldsymbol{r} \times(\boldsymbol{E} \times \boldsymbol{B}))=\int \mathrm{d}^{3} r(\boldsymbol{P} \times \boldsymbol{E}) .
$$

If we interpret that the left term is the rate of change of angular momentum, then for the $z$ component the result is

$$
-\int \mathrm{d}^{3} r \varepsilon_{0} \partial_{t}(\boldsymbol{r} \times(\boldsymbol{E} \times \boldsymbol{B}))_{z}=\int \mathrm{d}^{3} r(\boldsymbol{P} \times \boldsymbol{E})_{z}
$$

which is precisely the balance on which Beth's work is founded.

\section{3) Balance of angular momentum to solve Mansuripur's paradox}

Recently Mansuripur [10] contended that there is a violation of the principle of relativity if the Lorentz force density is applied to the interaction between a magnetic point dipole and a point charge. The paradox arises from the fact that while in the rest system there is not any torque between the dipole and the point charge, in a reference system moving with respect to the rest system a torque appears that constitutes a violation of the principle of relativity. To solve this paradox Mansuripur [10] proposes to use a more general force density, which is the one proposed by Einstein and Laub in 1908 [9], that is,

$$
\begin{aligned}
\left.\boldsymbol{f}\right|_{\text {Ein-Laub }}= & \rho \boldsymbol{E}+\mu_{0} \boldsymbol{J} \times \boldsymbol{H}+(\boldsymbol{P} \cdot \nabla) \boldsymbol{E}+\mu_{0}\left(\partial_{t} \boldsymbol{P}\right) \times \boldsymbol{H} \\
& -\varepsilon_{0} \mu_{0}\left(\partial_{t} \boldsymbol{M}\right) \times \boldsymbol{E}+\mu_{0}(\boldsymbol{M} \cdot \nabla) \boldsymbol{H} .
\end{aligned}
$$

Mansuripur [10] then states that "To guarantee the conservation of angular momentum Equation (61) must be supplemented with the following expression for the torque density exerted by electromagnetic fields on a material":

$$
\boldsymbol{\tau}=\boldsymbol{r} \times\left.\boldsymbol{f}\right|_{\text {Ein-Laub }}+\boldsymbol{P} \times \boldsymbol{E}+\mu_{0} \boldsymbol{M} \times \boldsymbol{H} .
$$


(He uses a different definition of the constitutive relation between the magnetic field and the magnetic induction. We use the standard definition, so there are some differences in $\mu_{0}$ factors). We can see that these torques are proposed with plausibility arguments founded on the Lorentz force law, while we offer a general and unified approach based on balance equations derived from the Maxwell equations.

Our approach let us to obtain, among others, these force and torque densities directly from the Maxwell equations. The respective balance equations are Equation (6) and Equation (37), which gives a sounder foundation to these proposals. Then instead of taking the Lorentz force density as a postulate independent of the macroscopic Maxwell equations, we derive from these several balance equations from which several force and torque densities emerge in a natural way, among them the usual Lorentz force density in the limit of a test charge in a vacuum. Since the Maxwell equations are intrinsically relativistic, there is no conflict with relativity.

A point that must be noted is that Masuripur [10] uses a macroscopic force density, that of Einstein and Laub [9], assuming its validity for point dipoles as given by

$$
\boldsymbol{P}=\boldsymbol{p} \delta\left(\boldsymbol{r}-\boldsymbol{r}^{\prime}\right)
$$

or

$$
\boldsymbol{M}=\boldsymbol{m} \delta\left(\boldsymbol{r}-\boldsymbol{r}^{\prime}\right)
$$

This is a delicate point that deserves further discussion, but is not essential for the argumentation here exposed. Our point is that the Lorentz force law is not independent from the Maxwell equations, but a particular case of the balance equations deduced from these equations.

\section{4) Open questions about these balance equations}

We have shown that the structure of the Maxwell equations can be transformed into the structure of momentum and angular momentum balance equations. However, we still do not consider dispersive media, or thermodynamic effects on electromagnetism. As an example of efforts in this direction we can mention Barnett's proposal about the flux of angular momentum [16]. Though he uses a transformation to Fourier's space, his results hold only for a vacuum. Thus his proposal does not permit to treat the experimental facts described by Beth [15]. We think our approach demonstrates that this experiment has its theoretical explication in the balance equations here derived.

\section{Conclusion}

In this work we have extended some electromagnetic linear momentum balance equations, to angular momentum balance equations, obtaining in this way different torque densities. In past works we have shown how the macroscopic Maxwell equations can be transformed into different balance equations for linear momentum. These balance equations imply different force densities that have as partic- 
ular or limit cases the Lorentz force density, the Helmholtz force density, or the Einstein-Laub [9] force density. In the same way we have shown here how from these balance equations we can derive corresponding balance equations for angular momentum. Thus these balance equations are implications of the Maxwell equations, and therefore as sound as these and the constitutive relations assumed. In this way they provide theoretical support to the experimental work of Beth [15], or permit to understand the paradox posed by Mansuripur [10]. Thus we have provided a unified and general theoretical frame to appraise some proposals of electromagnetic torques based on the Lorentz force law with plausibility arguments.

\section{References}

[1] Hirose, A. (2010) Radiation Pressure on a Dielectric Surface. Canadian Journal of Physics, 88, 247-252. https://doi.org/10.1139/P10-018

[2] Shvechenko, A. and Hoenders, B.J. (2010) Microscopic Derivation of Electromagnetic Force Density in Magnetic Dielectric Media. New Journal of Physics, 12, Article ID: 053020. https://doi.org/10.1088/1367-2630/12/5/053020

[3] Astrath, N.G.C., Malacarne, L.C., Baesso, M.L., Lukasievicz, G.V.B. and Bialbowski, S. (2014) Unravelling the Effects of Radiation Forces in Water. Nature Communications, 5, 4363. https://doi.org/10.1038/ncomms5363

[4] Brevik, I. and Ellingsen, A. (2010) Transverse Radiation Force in a Tailored Optical Fiber. Physical Review A, 81, Article ID: 011806. https://doi.org/10.1103/PhysRevA.81.011806

[5] Dodin, L.Y. and Fisch, N.J. (2012) Axiomatic Geometrical Optics, Abraham-Minkowski controversy, and Photon Properties Derived Classically. Physical Review A, 86, Article ID: 053834. https://doi.org/10.1103/PhysRevA.86.053834

[6] Ramos, T., Rubilar, G.F. and Obukhov, Y.N. (2015) First Principles Approach to the Abraham-Minkowski Controversy for the Momentum of Light in General Linear Non-Dispersive Media. Journal of Optics, 17, Article ID: 025611. https://doi.org/10.1088/2040-8978/17/2/025611

[7] Zhang, L., She, W., Peng, N. and Leonhardt, U. (2015) Experimental Evidence for Abraham Pressure of Light. New Journal of Physics, 17, Article ID: 053035. https://doi.org/10.1088/1367-2630/17/5/053035

[8] Brevik, I. (1979) Experiments in Phenomenological Electrodynamics and the Electromagnetic Energy-Momentum Tensor. Physics Reports, 52, 133-201. https://doi.org/10.1016/0370-1573(79)90074-7

[9] Einstein, A. and Laub, J (1908) Über die elektromagnetischen Grundgleichungen für bewegte Körper. Annals of Physics, 331, 532-540. https://doi.org/10.1002/andp.19083310806

[10] Mansuripur, M. (2012) Trouble with the Lorentz Law of Force: Incompatibility with Special Relativity and Momentum Conservation. Physical Review Letters, 108, Article ID: 193901. https://doi.org/10.1103/PhysRevLett.108.193901

[11] Jackson, J.D. (1999) Classical Electrodynamics. 3rd Edition, John Wiley and Sons, New York.

[12] Campos, I., Jiménez, J.L. and López-Mariño, M.A. (2012) Electromagnetic Momentum Balance Equation and the Force Density in Material Media. Revista Brasileira de Ensino de Física, 34, 2303.

[13] Jiménez, J.L., Campos, I. and López-Mariño, M.A. (2013) Several Energy-Momentum-Stress 
Balance Equations Deduced from Maxwell's Equations in Material Media. Non-Covariant and Explicitly Covariant Formulation. European Physical Journal-Plus, 128, 129. https://doi.org/10.1140/epjp/i2013-13129-6

[14] Poynting, J.H. (1909) The Wave Motion of a Revolving Shaft, and a Suggestion as to the Angular Momentum in a Beam of Circularly Polarised Light. Proceedings of the Royal Society A, 82, 560-567. https://doi.org/10.1098/rspa.1909.0060

[15] Beth, R.A. (1936) Mechanical Detection and Measurement of the Angular Momentum of Light. Physical Review, 50, 115-125. https://doi.org/10.1103/PhysRev.50.115

[16] Bohren, C.F. (2011) Radiation Forces and Torques without Stress (Tensors). European Journal of Physics, 32, 1515-1517. https://doi.org/10.1088/0143-0807/32/6/006

[17] Allen, L., Beijersbergen, M. W., Spreeuw, R. J. C. and Woederman, J. P. (1992) Orbital Angular Momentum of Light and the Transformation of Laguerre-Gaussian Laser Modes. Physical Review A, 45, 8185-8189. https://doi.org/10.1103/PhysRevA.45.8185

[18] Allen, L. and Padgett, M.J. (2000) The Poynting Vector in Laguerre-Gaussian Beams and the Interpretation of Their Angular Momentum Density. Optics Communications, 184, 67-71. https://doi.org/10.1016/S0030-4018(00)00960-3

[19] Lai, H.M. (1980) Electromagnetic Momentum in Static Fields and the Abraham-Minkowski Controversy. American Journal of Physics, 48, 658-659. https://doi.org/10.1119/1.12331

[20] Jiménez, J.L., Campos, I. and López-Mariño, M.A. (2011) Electromagnetic Momentum in Magnetic Media and the Abraham-Minkowski Controversy. European Journal of Physics, 32, 739-746. https://doi.org/10.1088/0143-0807/32/3/010

[21] Barnett, S.M. (2002) Optical Angular-Momentum Flux. Journal of Optics B: Quantum and Semiclassical Optics, 4, S7-S16. https://doi.org/10.1088/1464-4266/4/2/361

[22] Cameron, R.P., Speirits, F.C., Gilson, C.R., Allen, L. and Barnett, S.M. (2015) The Azimuthal Component of Poynting's Vector and the Angular Momentum of Light. Journal of Optics, 17, Article ID: 125610. https://doi.org/10.1088/2040-8978/17/12/125610

[23] Panofsky, W.K.H. and Phillips, M. (1962) Classical Electricity and Magnetism. Addison-Wesley, Reading. 